\title{
Power-law frequency distribution of $H / V$ spectral ratio of seismic signals: Evidence for a critical crust
}

\author{
Patrizio Signanini ${ }^{1}$ and Angelo De Santis ${ }^{2,1}$ \\ ${ }^{1}$ Università di Chieti e Pescara “G. D'Annunzio”, Dipartimento Geotecnologie per l'Ambiente e il Territorio, Chieti Scalo, Italy \\ ${ }^{2}$ Istituto Nazionale di Geofisica e Vulcanologia, Rome, Italy
}

(Received March 4, 2011; Revised July 30, 2011; Accepted August 5, 2011; Online published March 2, 2012)

\begin{abstract}
We analyse records from several seismic stations in Central Italy (including some with multiple recording sites) for the statistical properties of spectra of both noise and earthquake signals. The main result is that the power-law scaling of most of the spectral $H / V$ ratio statistical distributions leads to a so-called heavy tail. This is interpreted as a statistical distribution with a fractal dimension of about 2 , which is almost certainly caused by a porous percolating medium beneath the stations due to some universal property of the crustal rocks crossed by the elastic waves propagation suggesting that the uppermost crust is in a critical state.
\end{abstract}

Key words: Seismic signal, critical crust, $H / V$ spectral ratio, fractal dimension.

\section{Introduction}

Amongst the statistical distributions of physical phenomena, we can distinguish between wild and mild distributions according to the character of their extreme values, i.e. their distribution tails. In particular, the terms wild or mild are here related to the presence of heavy or thin tails, respectively, in the frequency-size distributions. Current usage of these terms regards the idea that heavy-tailed distributions are those that have power-law frequency-size distributions, and where not all moments are finite; these distributions arise from scale-invariant processes (Ghil et al., 2011). What is interesting is that distributions with a wild character, such as the power-law distributions, in contrast to those with mild ones, such as Gaussian distributions, are ubiquitous in many natural phenomena characterised by some criticality process (Sornette, 2006, 2009). This can be explained by the presence of their heavy tail that emerges from the non-linear coupling properties, when the probability of values far from the statistical mode (such as the occurrence of disasters with respect to normal situations) has still some chance of occurring. Thus, the presence of this heavy tail could be a sign of non-linear characteristics of the dynamical system of the quantities under investigation.

Statistical distributions of some quantities related to seismicity are of considerable importance for scientific studies either per se, or in order to assess reasonable seismic hazard.

The best known distribution is the Gutenberg-Richter relationship (Gutenberg and Richter, 1954) between the logarithm of the number of earthquakes $n$ in a given time with a

Copyright (C) The Society of Geomagnetism and Earth, Planetary and Space Sciences (SGEPSS); The Seismological Society of Japan; The Volcanological Society of Japan; The Geodetic Society of Japan; The Japanese Society for Planetary Sciences; TERRAPUB.

doi:10.5047/eps.2011.08.006 magnitude equal to, or greater than, $m$ :

$$
\log _{10} n=-b m+\log _{10} a
$$

where $a$ and $b$ are constants. $b$ is generally in the range $0.8<b<1.2$ (Wesnousky, 1999) and can be related to the regional strain over the area of concern (Smith, 1976) and to the entropy of earthquakes (De Santis et al., 2011). Equation (1) is a wild power-law distribution relating the number $n$ of earthquakes and the corresponding energy (or the characteristic size of the rupture), so that it can be interpreted in terms of the fractal dimension $D$ of the distributed seismicity with $D=2 b$ (Turcotte, 1997, page 59), that is $D \approx 2$.

Another known statistical relationship is the length distribution of crustal faults, usually described by a fractal (power law) distribution (Scholz et al., 1993) with a fractal dimension in the range 1.4 to 1.7 (Barton, 1985).

Statistical studies of the seismic signal per se can be more difficult. They are mainly based on seismometer data, and may be equipment-dependent. We attempt this approach, but try to avoid equipment-dependence.

It is well-established that seismic site response is related to the characteristics of the uppermost surface layers. Several techniques have been developed to estimate the seismic site response. Amongst these, Nakamura (1989) proposed a simple method to deduce the seismic site response from the spectral ratio of the horizontal $H$ and vertical $V$ components of microtremors (seismic noise). In principle, the ratio between two components eliminates seismometer transfer-functions, and provides an objective measure of the mechanical characteristics of the medium through which the seismic waves propagate and/or other information related to physical phenomena. Nakamura's spectral $(H / V)$ ratio technique has been largely applied to study site effects (Lermo and Chavez-Garcia, 1994; Field and Jacob, $1995)$ with the main objective of estimating the fundamental frequency response of soft deposits. This technique is 
Table 1. Details of sites of Italian seismic stations used in this study.

\begin{tabular}{|c|c|c|c|}
\hline Site & Longitude E & Latitude N & Geological characteristics \\
\hline 1. Fabriano (Borgo) & $12^{\circ} 53^{\prime} 55^{\prime \prime}$ & $43^{\circ} 20^{\prime} 10^{\prime \prime}$ & $\begin{array}{l}\text { Holocenic-Pleistocenic fluvial-colluvial deposits. } \\
\text { Marly Tertiary bedrock at } 10 \text { meters depth. }\end{array}$ \\
\hline 2. Celano 1 & $13^{\circ} 33^{\prime} 08^{\prime \prime}$ & $42^{\circ} 03^{\prime} 42^{\prime \prime}$ & $\begin{array}{l}\text { Holocenic-Pleistocenic fluvial and fluvioglacial deposits } \\
\text { (gravels and sands). Carbonatic Bedrock at 10-100 m. }\end{array}$ \\
\hline 3. Celano 2 & $13^{\circ} 31^{\prime} 54^{\prime \prime}$ & $42^{\circ} 00^{\prime} 04^{\prime \prime}$ & $\begin{array}{l}\text { Holocenic-Pleistocenic lacustrine deposits (clays, silts and sands). } \\
\text { The bedrock is at several hundred metres depth. }\end{array}$ \\
\hline 4. Celano 3 & $13^{\circ} 31^{\prime} 54^{\prime \prime}$ & $42^{\circ} 00^{\prime} 00^{\prime \prime}$ & $\begin{array}{l}\text { Holocenic-Pleistocenic lacustrine deposits (clays, silts and } \\
\text { sands). The bedrock is at several hundred metres depth. }\end{array}$ \\
\hline 5. Celano 4 & $13^{\circ} 31^{\prime} 54^{\prime \prime}$ & $41^{\circ} 55^{\prime} 45^{\prime \prime}$ & Outcropping Mesozoic bedrock (stratified limestone). \\
\hline 6. Celano 5 & $14^{\circ} 23^{\prime} 44^{\prime \prime}$ & $41^{\circ} 56^{\prime} 36^{\prime \prime}$ & Sub-outcropping Mesozoic bedrock (massive limestone). \\
\hline 7. Fabriano (Vallina) & $12^{\circ} 48^{\prime} 46^{\prime \prime}$ & $43^{\circ} 20^{\prime} 44^{\prime \prime}$ & $\begin{array}{l}\text { Holocenic detrital deposits: rubbles, pebbles, gravel with clay. } \\
\text { The depth of the bedrock is at about twenty meters. }\end{array}$ \\
\hline 8. Fabriano (Cupo) & $12^{\circ} 47^{\prime} 48^{\prime \prime}$ & $43^{\circ} 20^{\prime} 28^{\prime \prime}$ & $\begin{array}{l}\text { Holocenic detrital deposits: pebbles, gravel, sands with clays. } \\
\text { The depth of the bedrock is at about twenty meters. }\end{array}$ \\
\hline 9. Fabriano (Belvedere) & $12^{\circ} 51^{\prime} 42^{\prime \prime}$ & $43^{\circ} 14^{\prime} 18^{\prime \prime}$ & Outcropping Tertiary bedrock: marls, silty marls and calcarenites. \\
\hline 10. Fabriano (Spina) & $12^{\circ} 55^{\prime} 09^{\prime \prime}$ & $43^{\circ} 19^{\prime} 55^{\prime \prime}$ & $\begin{array}{l}\text { Holocenic eluvial-colluvial deposits: clays, limes and sands. } \\
\text { The Miocenic-Tertiary bedrock constituted by marly clays } \\
\text { is at } 3 \text { to } 10 \text { metres depth. }\end{array}$ \\
\hline 11. Raggiano & $13^{\circ} 2^{\prime} 4^{\prime \prime}$ & $43^{\circ} 8^{\prime} 24^{\prime \prime}$ & Marls \\
\hline 12. Caltabellotta & $13^{\circ} 12^{\prime} 50^{\prime \prime}$ & $37^{\circ} 34^{\prime} 40^{\prime \prime}$ & Carbonatic Bedrock \\
\hline
\end{tabular}

not wholly straightforward as it includes a filtering procedure that is not completely objective in that it requires an arbitrary choice of appropriate parameters. However, by analysing $H / V$ spectral ratios: (i) we avoid any conventional filtering in both time and frequency domains; on the other hand, (ii) we make some restrictions on the analysed data. In this short note, we do not deal with the aspects related to the strict interpretation of the spectral $H / V$ ratio (see, for instance, Fäh et al., 2001; Cara et al., 2003; Castellaro and Mulargia, 2009; Surve and Mohan, 2010; Tuan et al., 2011) but we analyse only its statistical frequency distribution for many seismometer digital segments where there appears only seismic noise, mainly produced by anthropic noise such as traffic, industry, etc. We study the heavy tail of the statistical distribution because we are particularly interested in the non-linear properties characterising the seismic phenomenon and/or the medium involved. We then extend this approach to some seismic events. This latter analysis has not been completely developed so only some general considerations can be made.

This paper is organised as follows: we describe the data collection, the related sites of observation, and then we analyse the data in terms of their statistical distribution. Finally, we interpret and discuss the results in terms of fractal properties.

\section{Data Collection}

The sites where the seismic stations were installed have been chosen for their different geological characteristics (Table 1). Sites 1-11 (at three seismic stations) are in Umbria and Marche (Central Italy), in the area shaken by the 1997 Umbria-Marche Earthquake Sequence (Amato et al., 1998; Chiaraluce et al., 2003; Miller et al., 2004; Antonioli et al., 2005), as a result of an initiative of the Dipartimento Geotecnologie per l'Ambiente e il Territorio of Chieti-Pescara University. Most of the seismic sites (1-
10) employ seismometers Willmore MARK III, with a minimum frequency sampling of $50 \mathrm{~Hz}$. At the Raggiano seismic station, a geophone MARK L4C-3D, with a sampling of $62.5 \mathrm{~Hz}$, was employed. Raggiano was installed as a result of the GNDT (Gruppo Nazionale Difesa dai Terremoti) Project. For comparison, a very-broad-band $(V B B)$ seismic station (sensor STS2 plus Quanterra datalogger) in Sicily (Caltabellotta) belonging to the INGV National Seismic Network has also been analysed. The latter station has been chosen for comparison with the other stations because it is in another seismogenetic part of Italy and has a wide spectral response sensor.

\section{Data Analysis and Results}

First, the three-component $(E, N$ and $V)$ digital data of the seismic noise from each seismic station listed in Table 1 have been fast-Fourier transformed (providing $\mathcal{F}[E], \mathcal{F}[N]$ and $\mathcal{F}[V]$, respectively, where $\mathcal{F}[X]$ indicates the operation of the Fourier transformation over the $X$ component). Second, we estimated the spectral $H$-component from $E$ and $N$, such as $\mathcal{F}[H]^{2}=\mathcal{F}[E]^{2}+\mathcal{F}[N]^{2}$ and computed the $\mathcal{F}[H]^{2} / \mathcal{F}[V]^{2}$ spectral ratio. In the following, we will refer to $\mathcal{F}[X]^{2}$ simply as $X$, meaning that it is derived from a fastFourier transformed operation over the component $X$, and squaring its value. Then, we estimated the statistical distributions for $H, V$ spectral components and the $H / V$ spectral ratio. Let $N(H / V)$ be the number of occurrences of a given ratio $H / V$ within $M$ samples; $p(x)=N(H / V) / M$ is the estimate of the probability of occurrence of a certain value $x$ of $H / V$ (the actual $p(x)$ would be with the limit of $M$ to infinity). The behaviour of $p(x)$ with $x$ is the statistical probability distribution. However, since we will investigate the scaling properties of the statistical distribution, we can simply analyse the behaviour of $N(H / V)$ with $H / V$.

Figure 1 shows the case of the station BEL (Belvedere di Fabriano, Central Italy; station n. 9 in Table 1). Here, 

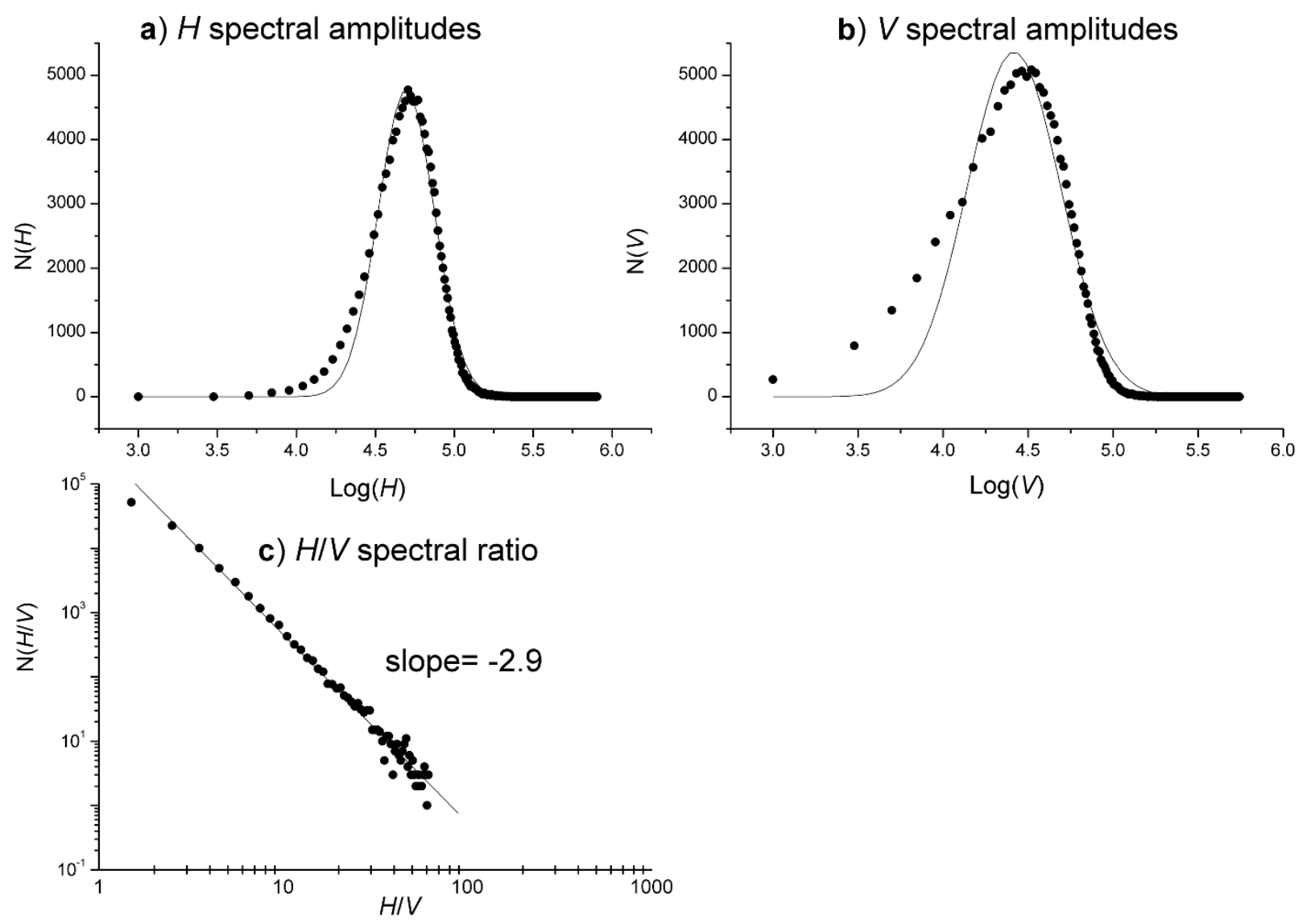

Fig. 1. Statistical distributions of seismic noise data from station Fabriano-Belvedere (station n. 9 in Table 1). In particular, the black circles are the distributions of: (a) $H$-component spectral amplitudes; (b) $V$-component spectral amplitudes; (c) $H / V$ spectral ratio. Continuous lines in (a) and (b) are the best-fit log-normal distributions, while in (c) it is the best-fit line.

we indicate the number of occurrences $N(H), N(V)$ and $N(H / V)$ of any given spectral amplitude or ratio value. Statistical distributions $N(H)$ are always practically lognormal distributions while $N(V)$ differs from this kind of distribution, mainly at lower values. It must be remembered that a log-normal distribution can be produced by the non-linear (multiplicative) interaction of some independent, identically distributed random variables. On the other hand, when we analyse the tail of the spectral ratio $H / V$ distribution, a very interesting feature arises. This part of the distribution shows a clear power-law scaling with an exponent of around -2.9 . Figure 2 (top) shows the analogous case of seismic noise recordings at Celano 3 (see Table 1), where the uppermost hundreds of meters are characterised by sedimentary deposits.

This scaling property of the spectral $H / V$ ratio distribution does not depend on the type of instrument used, nor on the kind of frequency distribution that has been analysed. Confirmation of this can be seen when the same analysis is applied to $V B B$ seismometer data from Caltabellotta (Sicily) as shown by Fig. 2 (bottom). Again, a line of slope -2.9 in the log-log distribution diagram closely follows most of the distribution.

The statistical analysis here has been performed with the specific objective of analysing the heavy tail of the distribution, in particular excluding those $H / V$ values smaller then or equal to 1 and greater than around 100 , allowing us to fo- cus the analysis over two orders of units. We also built the statistical distribution without considering data points after the first zero value. All this avoids considering the lowest values of both horizontal and vertical components, which could be critical being probably too close to the instrument resolution. The fact of having considered a band-limited $H / V$ should also avoid any aliasing effects, because one could expect that aliasing is usually transferred to small values of $H$ and $V$, so producing fictitious high or low values of $H / V$.

One could be led to believe that this power-law distribution is typical of only microtremors, that is of signals with no earthquake seismicity. On the other hand, we applied the same procedure to some seismic events of the UmbriaMarche (Central Italy) Earthquake Sequence of September and October, 1997. Figures 3 and 4 show the cases of earthquakes with magnitudes $M_{\mathrm{L}}=2.4$ and $M_{\mathrm{L}}=2.8$, respectively, as measured at the station of Raggiano for two events on the same day (18 October, 1997).

From these figures, the statistical distribution tail can still be fitted by a power law even during seismic activity, and the apparent slope of -2.9 is usually the same as that of the seismic noise data. Figure 4 also shows the best normal distribution (dashed curve) fitting the actual statistical data. The presence of the heavy tail is evident in the distribution with respect to the normal theoretical distribution, providing a power-law form of the descending right part of the 

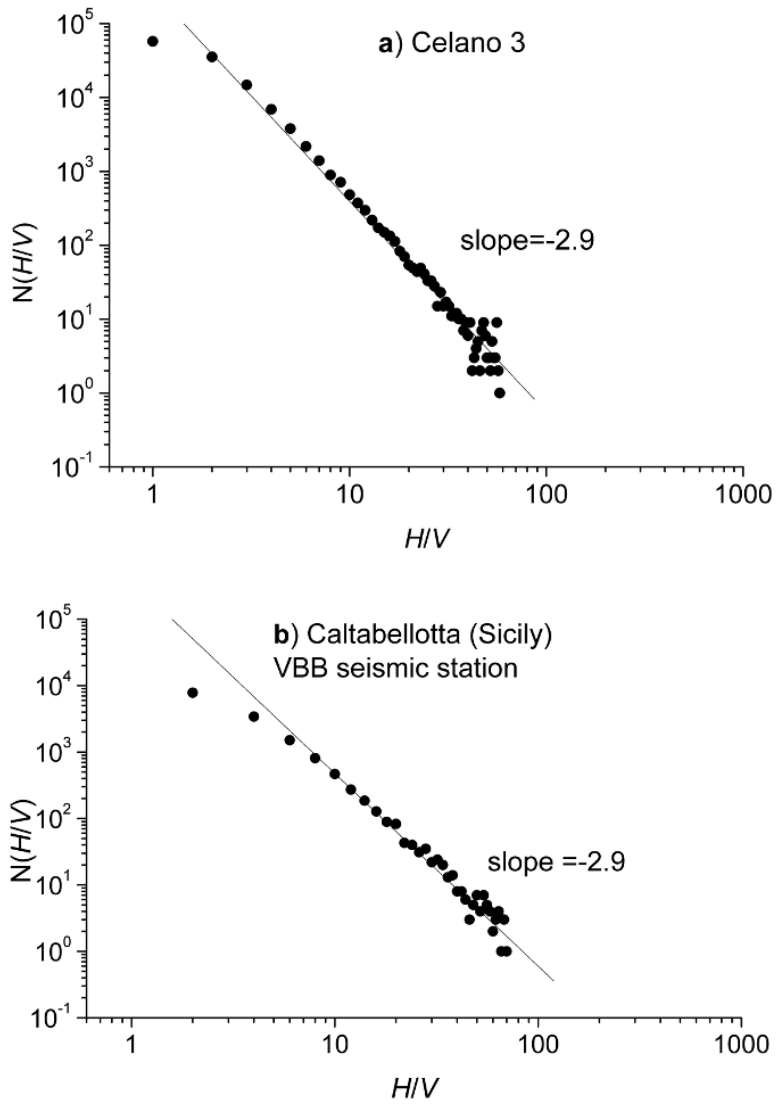

Fig. 2. Statistical distribution (black circles) of $H / V$ spectral ratio, i.e. $N(H / V)$, from: (a) Celano and from (b) the $V B B$ seismometer of Caltabellotta (Sicily, Italy) seismic noise data. Continuous lines in (a) and (b) are the best-fit linear regressions.

distribution. Actually, when fitting all points (with same weight) of the statistical distribution, slight differences of the slope arise (from a slope of -2.9 to a slope of -2.5 ), mostly due to the contribution of higher values of $H / V$ that tend to increase in occurrence with respect to no seismic activity. This part of the work is not yet completely investigated and will be the subject of further analyses.

\section{A Fractal Interpretation}

Let us recall that a power-law distribution $p(n)$ with a slope $-\alpha$ (i.e., $p(x) \sim x^{-\alpha}$ ) corresponds to a powerlaw cumulative distribution $\left(\int_{x}^{\infty} p\left(x^{\prime}\right) d x^{\prime}\right)$ with exponent $-\beta=-\alpha+1$ (the only exception is $\alpha=1$, when the cumulative distribution is logarithmic), in our case $\beta=1$.9. It is usually assumed that, when $\beta$ is between 0 and 3, the distribution can be considered fractal with a fractal dimension $D=\beta$ (Schroeder, 1991); however, the relation between $D$ and $\beta$ is often considered valid even outside this interval of $\beta$ (Turcotte, 1997, pages 16-17). In our case, this means that $D$ is close to 2 .

The $H / V$ spectral ratio could be, in principle, indicative of the characteristics of the medium through which the seismic waves propagate. The fact that the scaling exponent of most of the spectral ratio distribution is practically the same, either in seismic or aseismic activity, and independent of the recording location, could be interpreted as a more general signature of the propagation medium. This general aspect

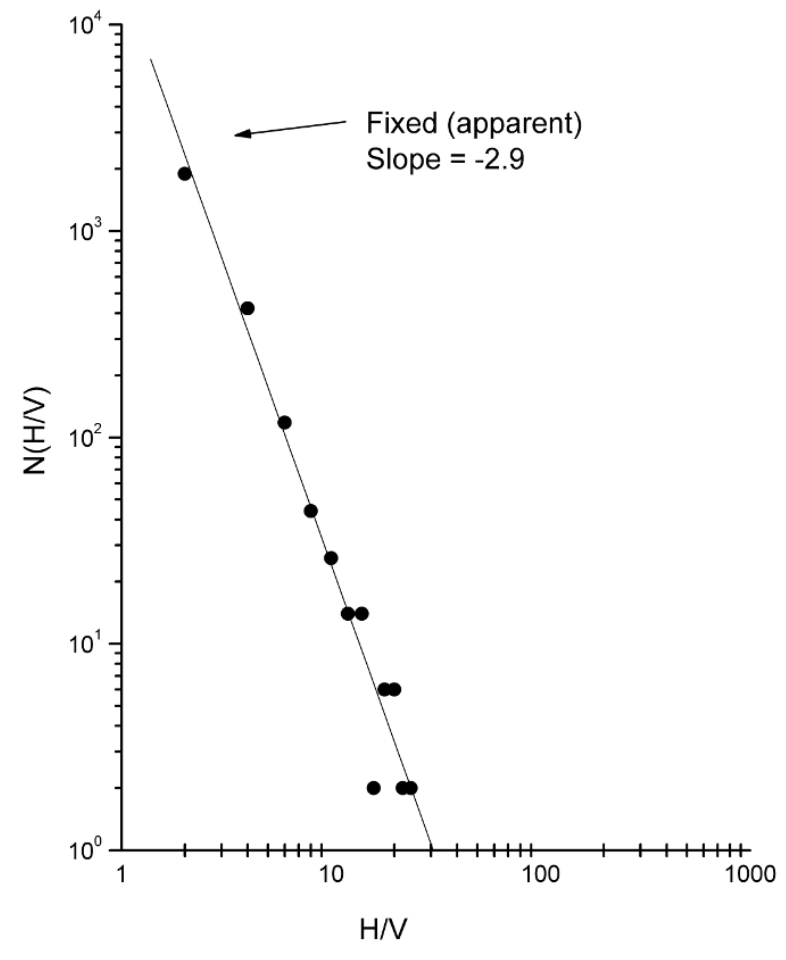

Fig. 3. Statistical distribution (black circles) of the $H / V$ spectral ratio, i.e. $N(H / V)$, at Raggiano Station for an earthquake of $M_{\mathrm{L}}=2.4$. Continuous line is the best-fit linear regression.

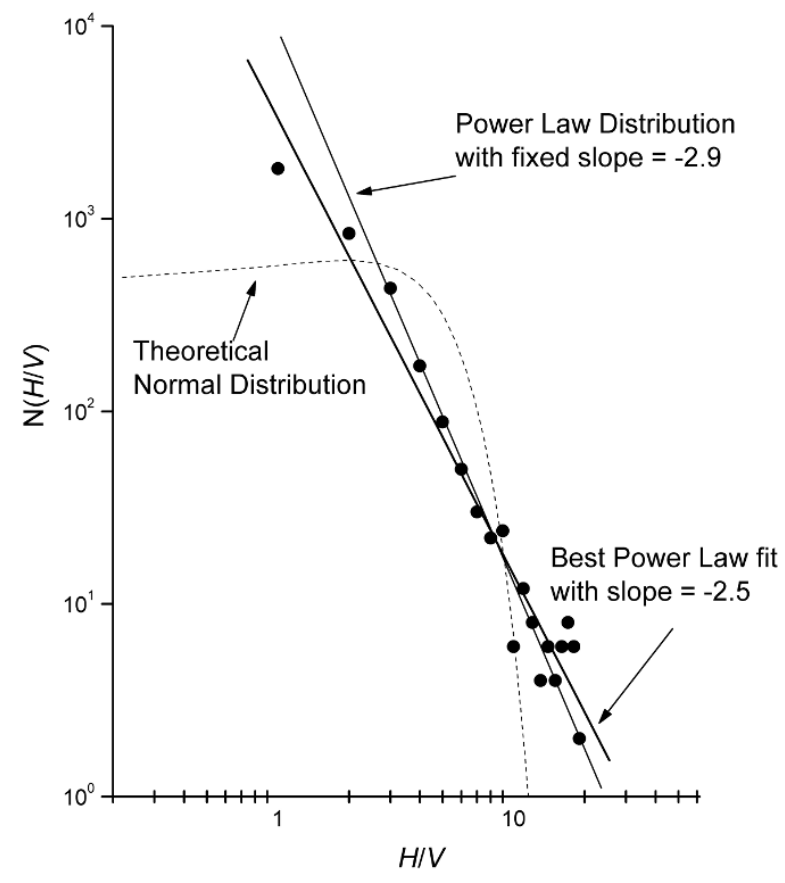

Fig. 4. Statistical distribution (black circles) of the $H / V$ spectral ratio, i.e. $N(H / V)$, at Raggiano Station for an earthquake of $M_{\mathrm{L}}=2.8$. Continuous lines are the best-fit linear regression (thick line) and the line with fixed slope $=-2.9$ (thin line). Dashed line is the theoretical normal distribution.

cannot be extracted by simply analysing the spectral ratio behaviour with frequency.

The fact that the statistical distribution tail of the spectral ratio $H / V$ has a power-law form also for some level of 
seismic activity (at least, this arises from the analysis of a few seismic events), would imply that the fractal properties are characterising not only the more superficial rocks but the whole crust. Morein et al. (1997) presented a simple 2D array of slider blocks connected by springs, interacting via static friction with a surface. Extending their findings to a 3D percolation model, would result in a fractal dimension similar to that found in our analysis, suggesting that the earthquakes form the foundation for a percolation cluster (Robertson et al., 1995).

Our results not only support this fact, but also add the important consideration that the $3 \mathrm{D}$ percolation model seems to persist even without earthquakes, that is without an explicit energy source, since our work concerns the whole seismic signal (noise and earthquake). Since the powerlaw distribution of percolation models is an indication of a critical state (e.g. Jensen, 1998), we argue that the $H / V$ power-law distribution is evidence that the uppermost crust is in a critical state (Bak and Tang, 1989; Bak, 1996; also Crampin, 2006). The value (near 2) of the fractal dimension in our analysis is close to that attributed to the distributed seismicity of the Gutenberg-Richter relationship (1); it is also the same as that found for the spatial distribution of earthquakes (Turcotte, 1997, chap. 4). We do not believe that these coincidences are fortuitous, but suggest that they arise naturally from the critical state of the rocks composing the uppermost crust.

\section{Conclusions}

Analysing the statistical distribution of the horizontalto-vertical spectral ratio of the seismic signal (both microtremors and seismic events), we find that the power-law cumulative distribution always has a scaling exponent of $\sim 2$. This result is not in contradiction with the use of the $H / V$ spectral ratio for isolating the resonance frequency of soft deposits: the latter application is just the interpretation of a point (or a small subset of points) of the whole statistical distribution. Where this particular distribution comes from is not an easy problem to solve. Power-law distributions are wild distributions that seem to characterise systems with very non-linear dynamics, or with stochastic multiplicative amplification effects (Sornette, 2006). Thus, seismic wave propagation, although apparently stochastic in each component of the seismic signal, is, in some way, organised in terms of distributions of the $H / V$ spectral ratio values. This organisation is probably indicative of the strong non-linear properties of rocks forming the uppermost crust, likely resulting from a critical state (Crampin, 2006). Crampin demonstrates that the criticality is caused by the distributions of fluid-saturated microcracks in virtually all rocks, being so closely-spaced that they verge on failure in fracturing and hence are critical-systems. To better exploit the characteristics of the $H / V$ statistical distributions with particular attention to the cases of increasing seismic activity, which have here been only marginally investigated, some further investigation is needed. This will possibly be the subject of a future work.

Acknowledgments. This work was initiated whilst ADS lectured as Contracted Professor of Geophysics at the University of Chieti.
We would like to thank our colleagues of the University of Chieti (UC) and INGV. Especially: Bruno Di Sabatino and Mario Rainone of UC, for their discussions and for providing a stimulating environment to play with this idea; Paolo Casale of INGV for providing the data from the $V B B$ station of Caltabellotta (Sicily, Italy); Paolo Favali and Patrizia Tosi of INGV for reading a preliminary version of the work and providing some interesting suggestions. We thank Stuart Crampin and an anonymous referee for their useful comments in reviewing the manuscript. The Raggiano data was taken from a CD-ROM of the executive project GNDT-CNR 1998, 6a1 (prepared by Govoni, A., Spallarossa, P. Augliera, L. Trojani; coordinator: M. Cattaneo). Part of this work was carried out under the SAGA-4-EPR Project, funded by the Italian Foreign Office. We dedicate this work to our colleague and beloved friend Bruno Di Sabatino who passed away in September 2010.

\section{References}

Amato, A., R. M. Azzara, C. Chiarabba, M. G. Giaccio, G. B. Cimini, M. Cocco, M. Di Bona, L. Margheriti, S. Mazza, F. Mele, G. Selvaggi, A. Basili, E. Boschi, F. Courboulex, A. Deschamps, S. Gaffet, G. Bittarelli, L. Chiaraluce, D. Piccinini, and M. Ripepe, The 1997 Umbria-Marche, Italy, earthquake sequence: A first look at the main shocks and aftershocks, Geophys. Res. Lett., 25(15), 2861-2864, 1998.

Antonioli, A., D. Piccinini, L. Chiaraluce, and M. Cocco, Fluid flow and seismicity pattern: Evidence from the 1997 Umbria-Marche (central Italy) seismic sequence, Geophys. Res. Lett., 32, L10311, doi: 10.1029/2004GL022256, 2005.

Bak, P., How the Nature Works, Springer-Verlag, New York, 1996.

Bak, P. and C. Tang, Earthquakes as self-organized critical phenomenon, J. Geophys. Res., 94, 15635-15637, 1989.

Barton, C. C., Fractal analysis of scaling and spatial clustering of fractures, in Fractals in the Earth Sciences, edited by Barton, C. C. and P. R. La Pointe, pp. 141-178, Plenum Press, New York, 1985.

Cara, F., G. Di Giulio, and A. Rovelli, A study on seismic noise variations at Colfiorito, Central Italy: Implications for the use of $H / V$ spectral ratios, Geophys. Res. Lett., 30(18), 1972, doi:10.1029/2003GL017807, 2003.

Castellaro, S. and F. Mulargia, The effect of velocity inversions on $H / V$, Pure Appl. Geophys., 166, 567-592, 2009.

Chiaraluce, L., W. L. Ellsworth, C. Chiarabba, and M. Cocco, Imaging the complexity of an active normal fault system: The 1997 Colfiorito (central Italy) case study, J. Geophys. Res., 108(B6), 2294, doi: 10.1029/2002JB002166, 2003.

Crampin, S., The new geophysics: a new understanding of fluid-rock deformation, in Eurock 2006: Multiphysics Coupling and Long Term Behaviour in Rock Mechanics, edited by Van Cotthem, A., R. Charlier, J.F. Thimus, and J.-P. Tshibangu, 539-544, Taylor and Francis, London, 2006.

De Santis, A., G. Cianchini, P. Favali, L. Beranzoli, and E. Boschi, The Gutenberg-Richter law and entropy of earthquakes: Two case studies in Central Italy, Bull. Seismol. Soc. Am., 2011 (in press).

Fäh, D., F. Kind, and D. Giardini, A theoretical investigation of average $H / V$ ratios, Geophys. J. Int., 145, 535-549, 2001.

Field, E. H. and K. H. Jacob, A comparison and test of various siteresponse estimation techniques, including three that are not referencesite dependent, Bull. Seismol. Soc. Am., 85(4), 1127-1143, 1995.

Ghil, M., P. Yiou, S. Hallegatte, B. D. Malamud, P. Naveaus, A. Soloviev, P. Friederichs, V. Keilis-Borok, D. Kondrashov, V. Kossobokov, O. Mestre, C. Nicolis, H. W. Rust, P. Shebalin, M. Vrac, A. Witt, and I. Zaliapin, Extreme events: Dynamics, statistics and prediction, Nonlin. Processes Geophys., 18, 295-350, 2011.

Gutenberg, B. and C. F. Richter, Frequency of earthquakes in California, Bull. Seismol. Soc. Am., 34, 185-188, 1954.

Jensen, H. J., Self-organised criticality: Emergent complex behaviour in physical and biologicl systems, in Lecture Notes in Physics, Cambridge Univ. Press, Cambridge, 1998.

Lermo, J. and F. J. Chavez-Garcia, Are microtremors useful for site response evaluation?, Bull. Seismol. Soc. Am., 84(5), 1350-1364, 1994.

Miller, S. A., C. Collettini, L. Chiaraluce, M. Cocco, M. R. Barchi, and J. B. Kraus, Aftershocks driven by a high-pressure $\mathrm{CO}_{2}$ source at depth, Nature, 427, 724-727, 2004.

Morein, G., D. L. Turcotte, and A. Gabrielov, On the statistical mechanics of distributed seismicity, Geophys. J. Int., 131, 552-558, 1997.

Nakamura, Y., A method for dynamic characteristics estimation of subsur- 
face using microtremor on the ground surface, QR Railway Tech. Res. Inst., 30(1), 25-33, 1989.

Robertson, M. C., G. G. Sammis, M. Sahimi, and A. J. Martin, Fractal analysis of 3-D spatial distributions of earthquakes with a percolation interpretation, J. Geophys. Res., 100, 609-620, 1995.

Scholz, C. H., N. H. Dawers, J.-Z. Yu, and M. H. Anders, Fault growth and fault scaling laws: Preliminary results, J. Geophys. Res., 98, 2195121961, 1993.

Schroeder, M., Fractals, Chaos, Power Laws: Minutes from an Infinite Paradise, pp. 429, W. H. Freeman, New York, 1991.

Smith, S. W., Determination of maximum earthquake magnitude, Geophys. Res. Lett., 3, 351-354, 1976.

Sornette, D., Critical Phenomena in Natural Sciences. Chaos, Fractals, Self-organization and Disorder: Concepts and Tools, 2nd edition, Springer series in Synergetics, Heidelberg, 2006.

Sornette, D., Probability distributions in complex systems, in Encyclopedia of Complexity and System Science, edited by R. A. Meyers, Springer Science, 2009.

Surve, G. and G. Mohan, Site response studies in Mumbai using $(H / V)$ Nakamura technique, Nat. Haz., 54, 783-795, 2010.

Tuan, T. T., F. Scherbaum, and P. G. Malischewsky, On the relationship of peaks and troughs of the ellipticity $(H / V)$ of Rayleigh waves and the transmission response of single layer over half-space models, Geophys. J. Int., 184, 793-800, 2011.

Turcotte, D. L., Fractals and Chaos in Geology and Geophysics, Cambridge Univ. Press, 1997.

Wesnousky, S. G., Crustal deformation processes and the stability of the Gutenberg-Richter relationship, Bull. Seismol. Soc. Am., 89(4), 11311137, 1999.

P. Signanini and A. De Santis (e-mail: angelo.desantis@ingv.it) 